\title{
EchoGéo
}

$20 \mid 2012$

Des mobilités aux transports. Regards croisés en Afrique de l'ouest

\section{Le chemin de fer Abidjan-Niger : la vocation d'une infrastructure en question}

Foussata Dagnogo, Olivier Ninot et Jean-Louis Chaléard

\section{OpenEdition}

Journals

Édition électronique

URL : https://journals.openedition.org/echogeo/13131

DOI : 10.4000/echogeo.13131

ISSN : 1963-1197

Éditeur

Pôle de recherche pour l'organisation et la diffusion de l'information géographique (CNRS UMR 8586)

Référence électronique

Foussata Dagnogo, Olivier Ninot et Jean-Louis Chaléard, « Le chemin de fer Abidjan-Niger : la vocation d'une infrastructure en question », EchoGéo [En ligne], 20 | 2012, mis en ligne le 13 juillet 2012,

consulté le 10 août 2021. URL : http://journals.openedition.org/echogeo/13131 ; DOI : https://doi.org/ 10.4000/echogeo.13131

Ce document a été généré automatiquement le 10 août 2021.

EchoGéo est mis à disposition selon les termes de la licence Creative Commons Attribution - Pas d'Utilisation Commerciale - Pas de Modification 4.0 International (CC BY-NC-ND) 


\title{
Le chemin de fer Abidjan-Niger : la vocation d'une infrastructure en question
}

\author{
Foussata Dagnogo, Olivier Ninot et Jean-Louis Chaléard
}

1 Comme ailleurs en Afrique subsaharienne, le chemin de fer a joué un rôle fondamental dans la pénétration, la pacification et l'exploitation économique de la Côte d'Ivoire sous la colonisation (Chaléard et al., 2006). Le chemin de fer «Abidjan-Niger ${ }^{1}$ », long de 1150 $\mathrm{km}$, qui relie Abidjan à l'actuel Burkina Faso, traversant le territoire ivoirien sur $630 \mathrm{~km}$ (carte 1), fut progressivement mis en place, accompagnant la mise en valeur de la colonie. Après les indépendances, la Côte d'Ivoire et le Burkina Faso décidèrent d'assurer ensemble la gestion de la ligne. Mais comme dans beaucoup d'autres États d'Afrique subsaharienne, le chemin de fer entra en déclin. En 1987, à la suite de désaccords entre les deux pays et en raison des difficultés du transport ferroviaire, les autorités ivoiriennes et burkinabés optèrent pour une gestion séparée. Cela ne résolut rien, bien au contraire: les problèmes s'accumulèrent et les déficits se creusèrent. Aussi, en 1995, sous la pression des bailleurs de fonds et dans un contexte de crise économique sévère, les deux États cédèrent-ils l'exploitation à un consortium privé, la Sitarail. Depuis septembre 1995, c'est cette société, composée majoritairement des groupes Bolloré et Maersk (tous deux présents dans les affaires maritimes), qui gère et exploite la ligne ferroviaire.

2 La vague de privatisation des chemins de fer en Afrique subsaharienne initiée dans les années 1990 a suscité de nombreux débats relatifs à la rentabilité et à l'efficacité de ce mode de transport sur le continent (De Brie, 2002 ; Proparco, 2011). Les opérations de privatisation ont elles-mêmes été discutées tant sur leurs modalités juridiques et financières que sur leur impact en matière d'organisation de l'espace et de développement aux échelles locales, nationales et supra nationales (Chaléard et al, 2006). Le cas de la privatisation du chemin de fer ivoiro-burkinabé nous permet d'alimenter ces débats à partir d'un cas précis. En effet, quinze ans après cette privatisation, de nombreuses interrogations se posent quant au rôle de la voie ferrée en 
Côte d'Ivoire. La privatisation devait permettre d'enrayer le déclin des trafics et de restaurer la situation financière du chemin de fer. Pour autant, même en cas de succès dans ces domaines, la cession au privé redonne-t-elle au chemin de fer le rôle qu'il a pu avoir à son âge d'or, au cours de la première moitié du $20^{\mathrm{e}}$ siècle ? La stratégie du groupe Bolloré, qui contrôle plus de la moitié des importations et exportations légales du Burkina Faso, se mesure à une échelle qui dépasse le cadre d'un État: il s'agit pour lui de développer un trafic marchandise international. Dans cette mesure, on peut se demander si le chemin de fer garde toujours son rôle structurant de l'espace ivoirien et s'il répond entièrement aux défis que doivent relever aujourd'hui les transports en Afrique de l'ouest : connexion de tous les lieux des territoires, intégration des espaces enclavés, articulation des transports locaux et internationaux par exemple (Lombard, Ninot, 2010).

Pour répondre à cette question, notre réflexion s'articulera autour de deux axes. Dans un premier temps, nous présenterons le rôle qu'a joué le chemin de fer dans la structuration de l'espace en Côte d'Ivoire depuis sa mise en place. Sans rouvrir les débats portant sur les effets structurants (Offner, 1993; Bavoux et al., 2005) ni ceux portant sur les liens entre transports et développement (Steck, 2009), il s'agira de montrer comment, dès son origine, le chemin de fer a fixé durablement les grandes lignes de l'organisation de l'espace ivoirien et fut marqué par une dialectique opposant son rôle national et son rôle international. Dans un second temps, nous évaluerons les conséquences de sa privatisation sur les différentes localités traversées, notamment celles dont les gares étaient autrefois régulièrement desservies. La priorité accordée au trafic international ne s'accompagne-t-elle pas d'une marginalisation des espaces traversés se traduisant par des impacts négatifs sur les économies locales?

\section{Le rôle du chemin de fer dans la structuration de l'espace ivoirien}

\section{Chemin de fer et organisation du territoire, de l'économie et de la logistique coloniale}

4 L'histoire du chemin de fer ivoirien commence avec la mission du commandant Houdaille au cours de l'année 1898, qui devait identifier une voie de pénétration vers l'intérieur de la colonie à partir de la côte. Si le tracé proposé fut accepté l'année suivante, le premier wharf de Grand Bassam ne fut installé qu'en 1901, en même temps qu'une autre mission (mission Avon) avait pour objectif d'identifier un port en eaux profondes. Le site d'Abidjan, sur la lagune Ebrié, fut retenu et l'on décida d'y fixer le point de départ de la future voie de pénétration ferroviaire. Une nouvelle mission fut confiée en 1903 à Crosson-Duplessis afin de fixer le tracé d'un premier tronçon de 79 $\mathrm{km}$, dont la construction fut lancée aussitôt (Maestri, 1976).

Malgré plusieurs obstacles et l'hostilité de certaines populations locales à ce projet (telle la révolte des Abé en 1909), la construction du chemin de fer continua. Il atteignit Agboville en 1907 puis Dimbokro en 1908 pour arriver trois ans plus tard à Bouaké, dont la gare fut inaugurée en 1912. Du fait de la Première Guerre mondiale en 1914, cette localité resta le terminus de la ligne pendant 11 ans. À la reprise des travaux en 1919, la création de la Haute Volta (actuel Burkina Faso) à partir de la division de la colonie du Haut Sénégal - Niger imposa une nouvelle conception et orientation du tracé afin de lui 
assurer un débouché maritime (Aloko, 2001). Après Katiola en 1924, le chemin de fer atteignit Ferkessédougou en 1929. Celle-ci fut préférée à Kong, grande cité précoloniale, jugée en perte de vitesse économique et commerciale. En 1933, le chemin de fer arriva à Bobo-Dioulasso, qui resta son terminus provisoire pendant deux décennies. Du fait de la Seconde Guerre mondiale, les travaux furent de nouveau interrompus et le chemin de fer atteignit enfin Ouagadougou (capitale de la Haute-Volta d'alors) le 23 octobre 1954.

6 La construction de la voie ferrée s'est accompagnée du développement d'un certain nombre de postes administratifs. C'est le cas par exemple du poste précédemment établi à Ery-Macouguié, qui fut transféré à Agboville après que le chemin de fer eut atteint cette localité en 1908. De même, le poste administratif au départ installé à $40 \mathrm{~km}$ de Dimbokro fut plus tard déplacé vers cette localité à l'arrivée des rails. En 1920, le village de Katiola fut érigé en poste administratif en prévision de l'arrivée du chemin de fer. Ce fut le cas également du village de Ferkessédougou, nouveau centre de commandement, en 1924 (Aloko, 2001).

7 Depuis les choix initiaux ayant guidé le projet colonial jusqu'à l'achèvement de l'infrastructure, se sont mises en place les bases d'une organisation territoriale, économique et logistique qui allait perdurer au-delà de la présence française.

\section{Le chemin de fer et l'essor des activités économiques}

8 Artère principale de l'économie de la colonie, le chemin de fer a été jusqu'en 1950 le moteur de développement socio-économique et spatial de la plupart des localités traversées (Chaléard, 2006). Agboville et Dimbokro lui doivent leur naissance tandis qu'Abidjan et Bouaké ont assis leurs statuts de pôles économiques du Nord et du Sud grâce à sa présence. Ainsi, tout le long de la ligne ferroviaire, des entreprises de transformation des produits du secteur primaire (quelques scieries à Abidjan, des stations d'égrenage de coton à vapeur à Dimbokro et à Bouaké, où fut aussi construite une usine de décorticage du riz, etc.) se sont installées et ont contribué à dynamiser les activités marchandes associées, accueillant, du coup, des commerçants européens, libanais et syriens (Maestri, 1976). Par exemple, dans la partie Sud de la Côte d'Ivoire, en l'absence de réseau routier fiable, toutes les productions des localités environnantes comme Bongouanou, Daoukro, Kotobi, Mbatto et Daoukro convergeaient vers les gares d'Abgoville et de Dimbokro. Ces deux localités connurent ainsi un développement spectaculaire grâce au chemin de fer avant de tomber en déclin à partir des années 1950, du fait de la concurrence de la route et de la crise locale de l'économie de plantation, dont le cœur de la production en Côte d'Ivoire se déplaçait plus à l'ouest (Bredeloup, 1989).

9 Ainsi, dans les premières années du rail, la ville de Dimbokro représentait un point de passage obligé entre la capitale et le nord du pays. La gare était un lieu de groupage et de dégroupage des marchandises. En 1912, Dimbokro était la première gare de l'intérieur exportatrice de kola, de graine et huile de palme, et de cuir. Elle occupait la seconde position pour l'expédition de maïs et de coton. À partir de 1915, la gare de cette localité accrut son aire d'influence en drainant les produits du Haut-Sassandra et de Séguéla. Pour mieux exporter le café et le cacao du cercle du Nzi-Comoé, les pistes formaient une route de desserte circulaire reliant les zones productrices au centre de distribution de Dimbokro. Cette route en circuit fermée fut appelée la «boucle du cacao ». Servant de lieu de rassemblement, de stockage, de transit, de redistribution et 
aussi de point d'accès à la région, Dimbokro devint le centre de cette boucle, le point de convergence unique de toute une région. La ville-gare contribua ainsi à la création d'une région de plantation et favorisa la richesse de l'arrière-pays.

Quant à Agboville, le cacao y prit de l'importance dans les années 1920. Cet essor fut favorisé par la proximité du chemin de fer, qui garantissait à l'époque aux producteurs des environs de meilleurs prix que ceux que pouvaient espérer les populations éloignées des gares ferroviaires (Chaléard, 2006). Des voies de communication partaient en étoile d'Agboville et reliaient la gare aux différents points du cercle (aujourd'hui département).

11 À partir de 1930, le café, d'abord exploité près de la voie ferrée, puis le long des pistes reliées aux gares, compléta l'économie de plantation locale et renforça la centralité de Dimbokro et d'Agboville jusque dans les années 1950, où la concurrence de la route et le transfert rapide de la production cacaoyère ivoirienne vers l'ouest provoquèrent leur déclin (Bredeloup, 1989 ; Chaléard, 2006).

12 Après l'indépendance en 1960, le chemin de fer, sous la direction de la Régie Abidjan Niger (RAN) gérée par les autorités ivoiriennes et burkinabés, maintint et renforça sa vocation principale de transporter les matières premières vers le port d'Abidjan (tableau 1).

Tableau 1- Exportations par voie ferrée (milliers de tonnes)

\begin{tabular}{|l|l|l|l|l|l|l|l|}
\hline & 1956 & 1957 & 1958 & 1959 & 1960 & 1961 & 1962 \\
\hline Bois & 67,7 & 54 & 64,6 & 68 & 77,3 & 60,6 & 34,8 \\
\hline Arachides & 2,5 & 2,3 & 3 & 3,8 & 2,1 & 16,2 & 29,7 \\
\hline Bananes & 6,8 & 7,5 & 7,5 & 3,9 & 5,9 & 6,1 & 8,4 \\
\hline Café-cacao & 30,4 & 19,7 & 19,3 & 24,5 & 21,1 & 35,6 & 16,6 \\
\hline Total & 107,4 & 83,5 & 94,4 & 100,2 & 106,4 & 118,5 & 89,6 \\
\hline
\end{tabular}

Source : Sitarail, Nouvelles du réseau, n86/87, 2004.

En complément, pendant la phase de gestion publique du chemin de fer (1960- 1994), l'insuffisance des infrastructures routières justifia qu'une attention particulière fut portée au transport de voyageurs. Le parc roulant comprenait à l'époque des trains rapides, des express et des omnibus. On pouvait compter 20 liaisons par jour dans les deux sens : Abidjan-Dimbokro, Abidjan-Bouaké, Abidjan-Ferkessédougou, AbidjanBobo-Dioulasso, Abidjan-Ouagadougou, Dimbokro-Bouaké, Bouaké-Bobo-Dioulasso, Bouaké-Ouagadougou, Ferkessédougou-Bobo-Dioulasso, Ferkessédougou-Ouagadougou, Ouagadougou-Bobo-Dioulasso. Le transport de personnes était une activité très importante pour la RAN. Selon les statistiques du Plan Transport (1988), sa part dans le chiffre d'affaires était de $37 \%$ en 1967, avec 2,6 millions de voyageurs, $52 \%$ en 1978, avec 4 millions de voyageurs, et 56,7\% en 1979, avec 3,8 millions de voyageurs (Yoboué et al., 1996). Toutes les localités traversées par le chemin de fer bénéficiaient au moins d'un temps d'arrêt des trains de voyageurs (cf. carte 1). Les populations en profitaient 
pour faire du commerce, ce qui contribuait à la redistribution des produits (vivriers notamment) et à la dynamisation des économies productives locales. Ainsi, plusieurs localités bénéficiant d'une gare ont vu leur économie se spécialiser dans certains produits destinés à Abidjan, comme Yapo (viande de brousse), Céchi (oignon) et Rubino (légumes) (Chaléard, 2006).

Carte 1 - Le chemin de fer Abidjan-Niger au cœur des territoires ivoirien et burkinabé

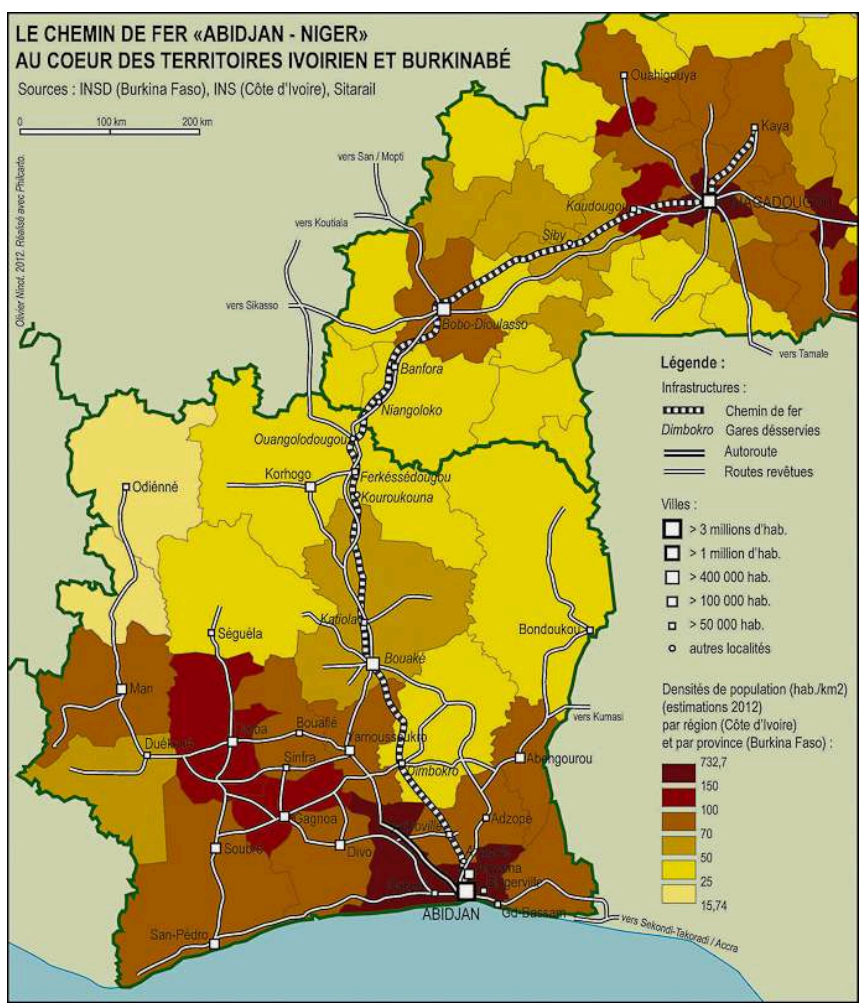

Source: INSD (Burkina Faso), INS (Côte d'Ivoire), Sitarail. Réalisation O. Ninot, 2012.

\section{La privatisation pour le maintien de l'exploitation du rail}

Comme la plupart des entreprises de chemin de fer de l'Afrique de l'ouest francophone, la RAN a connu une dégradation de sa situation économique et financière à partir des années 1980 malgré quelques efforts d'investissement et de modernisation du service de voyageurs consentis au cours des années 1970. Confronté à la concurrence de la route, à la mauvaise gestion et à l'insuffisance des subventions accordées par les États ivoirien et burkinabé, le transport ferroviaire a vu ses parts de trafic chuter progressivement. La gestion du chemin de fer Abidjan-Niger est devenue un poids non négligeable pour les finances publiques des deux États. Face à cette situation, les bailleurs de fonds ont plaidé pour une séparation entre l'infrastructure et l'exploitation, allant jusqu'à conditionner leur aide à cette option (Peguy, 1999). Se trouvant dans une situation de crise économique sévère, la Côte d'Ivoire et le Burkina Faso suivirent les recommandations du FMI et de la Banque mondiale. En 1992, les deux pays décidèrent ainsi de concéder l'exploitation du chemin de fer à une entreprise privée. À la suite d'un appel d'offres lancé à la fin de l'année 1993, c'est la Sitarail (Société Internationale de Transport Africain par Rail) qui fut retenue comme adjudicataire. Dans ce consortium, la SAGA (Société Anonyme de Gérance et 
Armement), l'une des filiales du groupe Bolloré spécialisée dans le transport de fret, a 32,65 \% des actions. Les États de Côte d'Ivoire et du Burkina Faso en conservent chacun $15 \%$. Les entreprises transnationales et locales Maersk, SICC, Transurb et Sofrerail en détiennent quant à elles environ $18 \%$. Enfin, la Bourse d'Abidjan en possède $16 \%$ et le personnel $3 \%$ (Martin, Micoud, 1998). Après de longues négociations, la privatisation de l'exploitation devint définitive en mars 1994 sous la forme de concession. La longueur des négociations s'explique en partie par la difficulté à faire converger des intérêts différents. L'enjeu pour les États ivoirien et burkinabé était d'assurer le maintien d'un service de transport ferroviaire. Pour les actionnaires privés de la Sitarail, il s'agissait de s'assurer de bénéficier des conditions leur permettant d'exercer une activité rentable. Pour cet objectif, l'héritage de la RAN était à la fois riche de potentialités et lourd de risques: une infrastructure et un parc roulant en partie vétustes, certaines des dessertes existantes peu rentables. La durée initiale de la concession fut fixée à quinze ans. Durant cette période, les parties se réunirent tous les cinq ans pour décider s'il y avait lieu de prolonger de cinq ans la concession au-delà de la date prévue de son expiration. Finalement, la convention de concession qui devait prendre fin en 2010 fut prolongée jusqu'en 2030 du fait de la crise politico-militaire en Côte d'Ivoire qui a entrainé de nombreuses pertes pour la Sitarail avec, notamment, une interruption du trafic de septembre 2002 à mai 2003.

$\mathrm{Si}$, dans la convention de concession, le transport ferroviaire est considéré comme une activité publique, le texte fait une distinction très claire entre les services de transport ferroviaire à caractère commercial et les services exploités à titre d'obligation de service public. Dans le premier cas, la Sitarail est libre de définir, selon ses propres critères de rentabilité, la configuration des services offerts. Elle est juste tenue d'informer les pouvoirs publics des critères utilisés pour considérer qu'un service est à caractère commercial. Les services exploités au titre d'obligation de service public peuvent être mis en œuvre à la demande d'un des États ou encore d'une collectivité locale aux termes d'un contrat spécifique passé entre les intéressés et Sitarail. Pour cela, des compensations doivent être versées à Sitarail, les caractéristiques du service et les modalités de ces compensations financières étant définies dans le contrat. Sitarail paye aux sociétés de patrimoine qui représentent les deux États une redevance de concession constituée de trois composantes: le droit d'usage, le loyer du matériel ferroviaire et le service de la dette contractée par les deux États pour financer les investissements de réhabilitation lors de la privatisation. La Sitarail paye également des impôts, des taxes et des contributions sociales, ce qui n'était pas le cas dans le cadre de la gestion publique. Après avoir été exonérée de redevance pour la première année d'exploitation, le montant de celle-ci fut fixé à $2 \%$ du chiffre d'affaires et à $4 \%$ dès la troisième année. Enfin, les États contractent les emprunts que Sitarail rembourse en totalité (Convention de concession de l'Abidjan-Niger, 1994).

Toutes ces mesures avaient pour but de mieux gérer et de rentabiliser le chemin de fer Abidjan-Niger. Mais les divergences d'intérêts sont vite apparues. D'un côté, si au début de la concession, la Sitarail a accepté la formule de remboursement des emprunts ferroviaires, elle semble la remettre en cause aujourd'hui et souhaite une révision de cet article. En effet, la société trouve injuste de devoir rembourser ces emprunts alors qu'elle n'est pas propriétaire du matériel ferroviaire ${ }^{2}$. D'un autre côté, elle a peu à peu réduit son engagement dans les dessertes répondant à une mission de service public 
(transport de voyageurs) pour se concentrer sur la partie rentable de son activité, le transport de marchandises sur de longues distances.

\section{Évolution du rôle du chemin de fer dans le contexte de gestion privée}

Depuis la privatisation de l'exploitation du chemin de fer Abidjan-Niger, le trafic de marchandises en transit, en dehors des périodes de troubles politiques graves, a connu une croissance remarquable. Cependant, ce succès a ses revers: au nom de la rentabilité économique, le concessionnaire a réduit la desserte de transport de voyageurs en fermant plusieurs gares dans des petites localités. La priorité donnée au trafic longue distance (principalement le transit) a alors de lourdes conséquences sur les économies locales.

\section{Une croissance continue du trafic de marchandises en transit}

Depuis la privatisation du chemin de fer, la Sitarail a mis l'accent sur le trafic de marchandises en transit, qui constitue en moyenne $80 \%$ de son chiffre d'affaires ${ }^{3}$ (en progression de 22 à 27 milliards de Francs CFA pour le fret entre 2006 et 2009). Les principales marchandises transportées de la Côte d'Ivoire vers le Burkina Faso sont le clinker, le ciment, les céréales, les conteneurs, les hydrocarbures, l'engrais. À la descente (du Burkina Faso vers la Côte d'Ivoire), ce sont en général les animaux vivants, le coton, les noix de karité, le sésame, les légumes, les fruits et le manganèse qui sont déchargés. Mise à part la période 2002-2003 au cours de laquelle la Côte d'Ivoire était en prise à un conflit armé, le transport ferroviaire de marchandises atteint, depuis la privatisation, des valeurs annuelles supérieures à ce qu'il était au début des années 1980, soit entre 800000 et 1000000 de tonnes $^{4}$ (graphique 1), en dépit de la partition de la Côte d'Ivoire jusque 2011. Mais ce trafic ne concerne que quelques gares ferroviaires. En Côte d'Ivoire, les principales localités desservies sont Abidjan et dans une moindre mesure Ferkessédougou et Ouangolodougou. Au Burkina Faso, ce sont essentiellement les gares de Ouagadougou, de Bobo-Dioulasso, de Banfora et de Koudougou. Parmi les 66 gares et haltes du chemin de fer, le trafic de marchandises ne concerne réellement que huit gares, si l'on ajoute la station de Bouaké, dont la desserte fut plusieurs fois stoppée en raison du conflit mais où un port sec fut néanmoins installé pour les produits à destination du Mali (Tape Bidi, 2010). Fonctionnant autrefois comme un corridor de transport irrigant et drainant les marchandises sur le territoire des deux pays, le chemin de fer fonctionne plutôt aujourd'hui comme un tunnel qui relie quelques centres urbains au port d'Abidjan (graphique 2). 
Graphique 1 - Evolution du trafic de marchandises ferroviaires selon les modes de gestion de 1980 à 2009

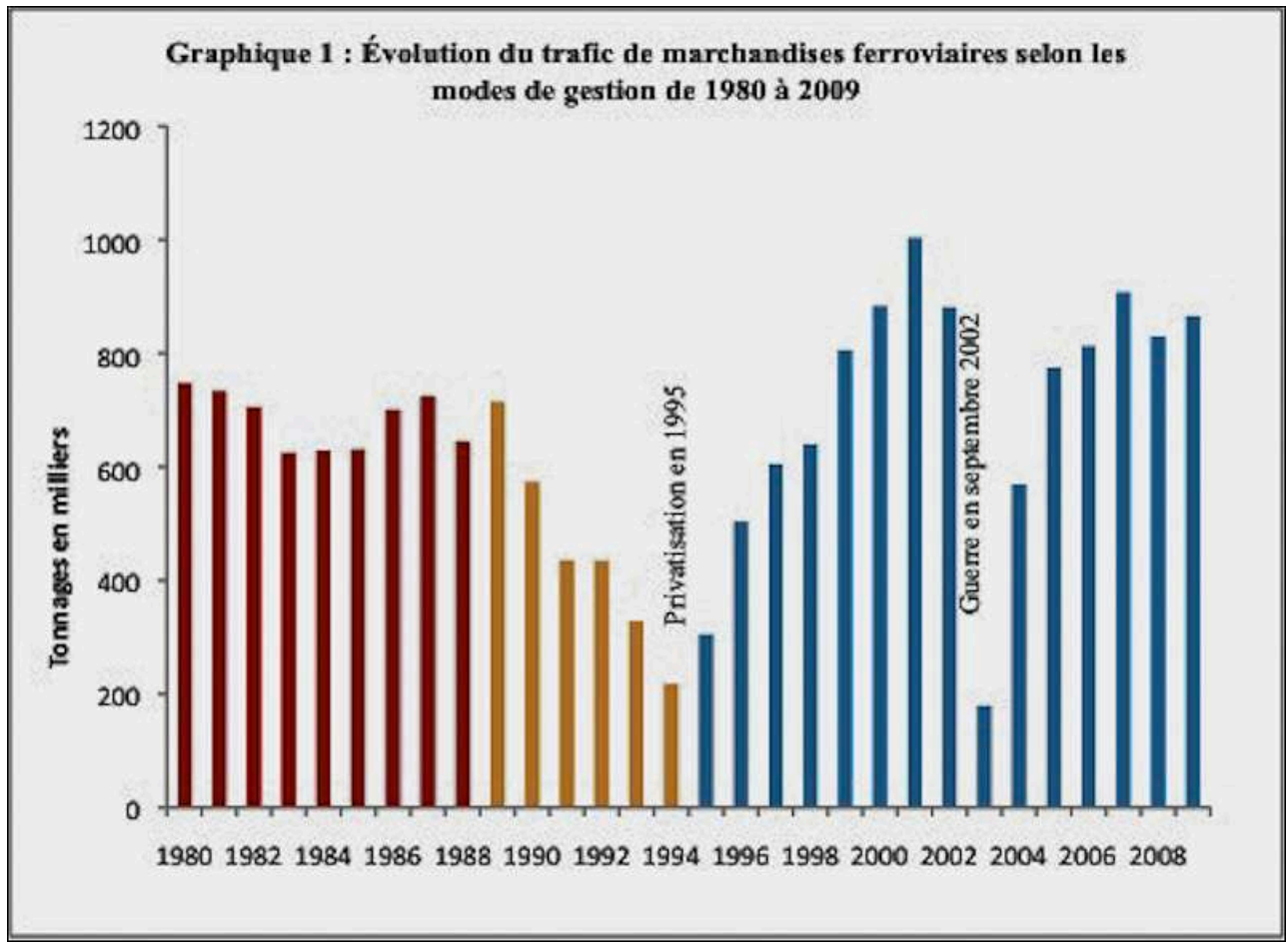

Source : Sitarail.

Graphique 2 - Importance du trafic en transit dans le total du fret en 2009

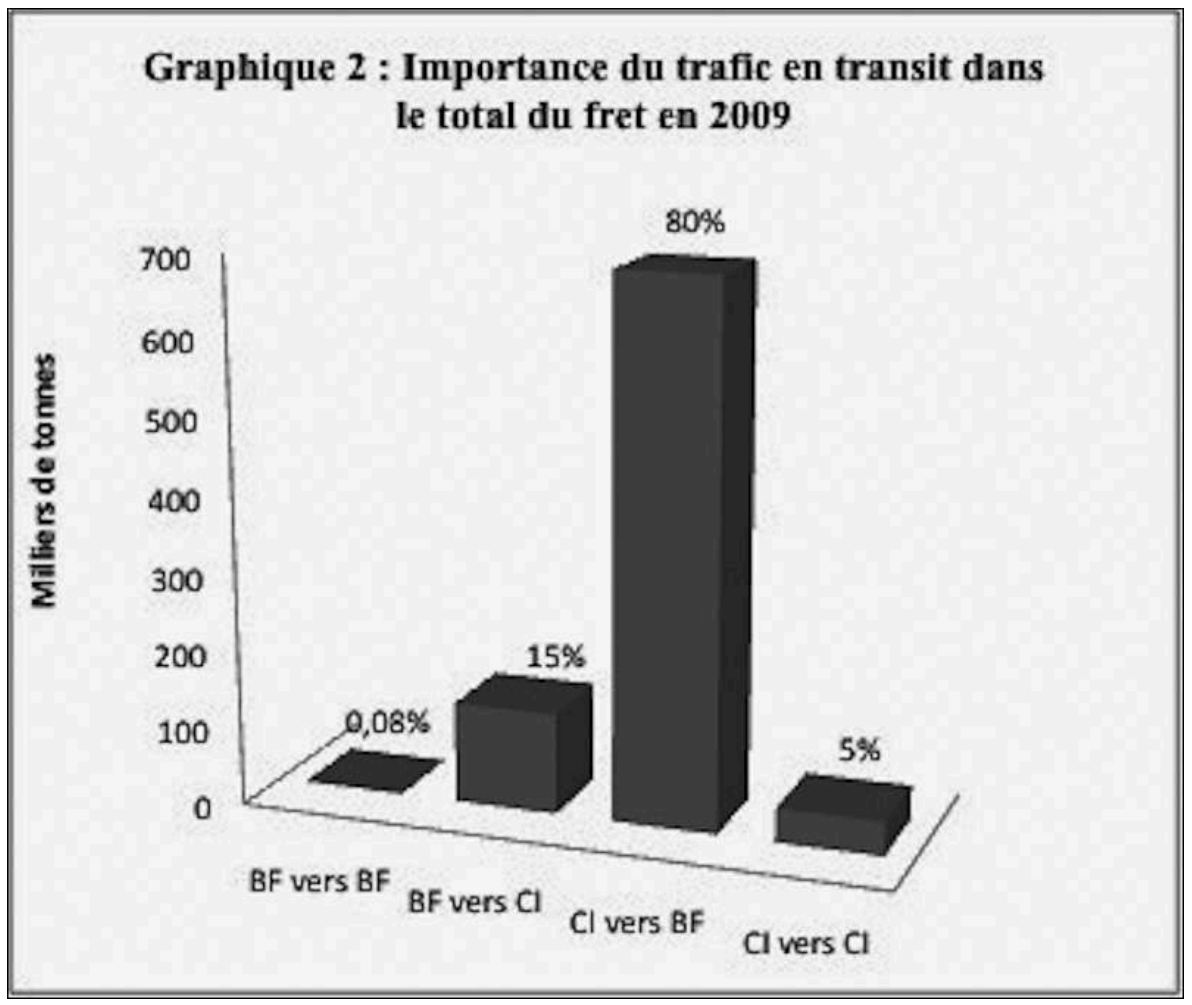

Source : Sitarail. 

commerciale de la Sitarail : 95 \% des trafics se font entre Abidjan et le Burkina Faso. Les statistiques prévisionnelles concernant le transport de marchandises, élaborées lors de la privatisation en 1995, ont été atteintes et même dépassées, avec une hausse d'environ $20 \%$ du trafic jusqu'au déclenchement de la guerre en 2002. L'ouverture récente des ports secs de Ferkessédougou (2008) et de Ouagadougou (2009) pour accueillir et traiter les marchandises à destination du Mali et du Niger a contribué à renforcer l'effet tunnel du chemin de fer et à consolider davantage la position de la Sitarail dans le transport des produits en transit, l'acheminement ferroviaire bénéficiant ainsi d'un avantage supplémentaire par rapport au transport routier.

\section{Marchandises versus voyageurs : au-delà des choix de la Sitarail, les intérêts d'un groupe puissant}

Acteur majeur de la logistique au niveau mondial, le groupe Bolloré a appliqué pour la Sitarail, dont il est l'actionnaire majoritaire, la stratégie qui lui a permis de maîtriser un dispositif logistique adossé sur les ports couvrant l'ensemble de l'Afrique de l'ouest. En premier lieu, par un jeu d'acquisitions successives (De Brie, De Guio, 2004), le groupe s'est retrouvé en charge de la quasi totalité des opérations logistiques depuis le transport maritime jusque l'acheminement final. Ensuite, en concentrant son offre de service sur le transport de marchandises, le groupe (à travers la Sitarail) s'est donné les moyens d'améliorer la qualité des dessertes proposées tant en termes de sécurité, de rapidité, que de prix. Ainsi, J. Debrie et S. De Guio (2004) interprètent cette stratégie comme une double adaptation du groupe au contexte ouest-africain. Une première consiste en l'intégration des différentes fonctions de transport, du maritime au terrestre. Une seconde est la recherche d'une situation de monopole ou d'hégémonie sur le marché du transport de marchandises longue distance, principalement de transit. Pour les auteurs, « ce ne sont pas la Côte d'Ivoire, le Togo, le Bénin, le Mali ou le Burkina Faso qui intéressent les opérateurs desservant les ports d'Abidjan, de Lomé ou de Cotonou, mais des espaces faits de transport de café, de coton, de cacao » (Debrie, De Guio, 2004).

Par conséquent, le transport de personnes n'apparaît pas comme une priorité pour la Sitarail et il a effectivement considérablement chuté depuis la privatisation de la gestion du chemin de fer (graphique 3). Déjà, au moment de la concession, la Sitarail avait jugé le matériel dédié au transport de voyageurs insuffisant et trop dégradé pour pouvoir faire face à la concurrence routière. Selon le concessionnaire, le matériel ne répondait pas aux normes minimales des trains de voyageurs et elle ne voulait donc pas de ce marché de transport. Ce n'est que sur l'insistance des autorités ivoiriennes et burkinabés que ce service a finalement été intégré dans les charges de la société5. Cependant, la Sitarail ne retint que les 2 trains express quotidiens (un dans chaque sens) pour assurer la liaison entre la Côte d'Ivoire et le Burkina Faso. Le train mixte hebdomadaire qui reliait Ouagadougou à Kaya fut vite supprimé (en 1998) en raison de la faiblesse du taux d'occupation des wagons (moins de $15 \%$ selon la Sitarail). En 2003, alors que la guerre interdisait la circulation des trains internationaux, la Sitarail mit pourtant en circulation un omnibus entre Abidjan et Dimbokro pour assurer le transport des personnes et surtout des produits vivriers dans cette zone où le réseau routier est dégradé. Mais au $1^{\mathrm{er}}$ avril 2004, la gestion des recettes des trains express et 
de l'omnibus fut confiée à un gestionnaire local, devenu la STIB (Société de Transport Ivoiro-Burkinabé) en 2006. Le désengagement de la Sitarail du transport de voyageurs s'est soldé par une diminution de la desserte proposée. Aujourd'hui, l'express ne circule qu'un jour sur deux et ne s'arrête que dans les localités urbaines.

Graphique 3 - Evolution du traffic de voyageurs de 1980 à 2003

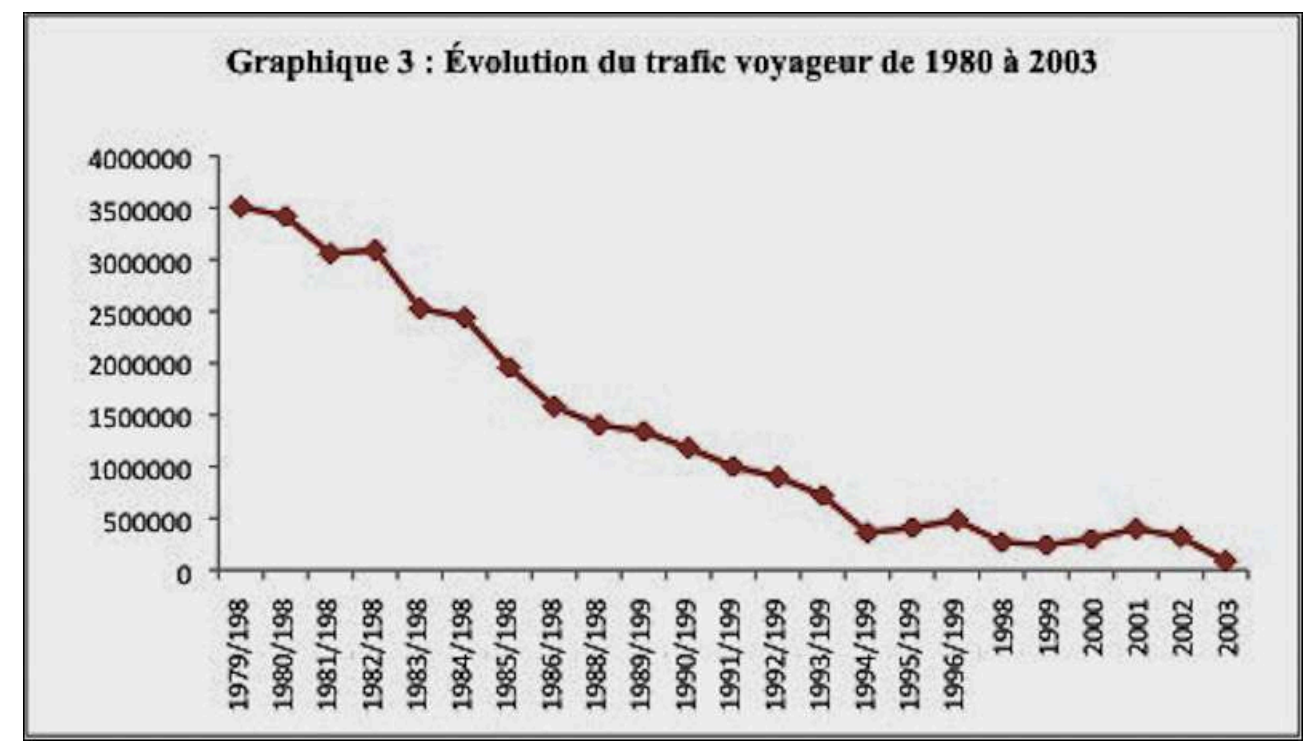

Source : Plan transport, 1988 ; données RAN et données Sitarail.

Quant à l'omnibus, le service a été suspendu fin 2010 à la suite des tensions postélectorales puis supprimé début 2011 pour cause d'insuffisance de matériel ${ }^{6}$. Pourtant, la circulation de ce train représentait une réelle opportunité pour les ruraux, car il desservait toutes les petites localités situées sur la ligne ( $c f$. carte 2). Les habitants de ces villages profitaient ainsi de la venue des commerçantes d'Abidjan pour vendre leurs produits agricoles. Ces femmes ${ }^{7}$ quittaient Abidjan avec l'omnibus pour se rendre dans les villages et les hameaux à proximité des rails afin d'y acquérir des produits vivriers auprès des paysannes. Au retour, le lendemain, elles chargeaient à chaque halte leurs achats qu'elles revendaient dans les gares situées entre Agboville et Abidjan, contribuant ainsi à la collecte et à la redistribution des produits agricoles locaux, tout comme à l'approvisionnement de la capitale. Les denrées transportées étaient principalement du riz, des avocats, des graines de palme, des bananes, des aubergines, des gombos, etc., mais aussi du charbon et dans une moindre mesure du gibier. La production des produits vivriers était devenue l'une des activités principales des localités rurales situées sur ce tronçon du chemin de fer, structuré et dynamisé par le chapelet de petites gares. 
Carte 2 - Le train omnibus, une desserte ferroviaire locale

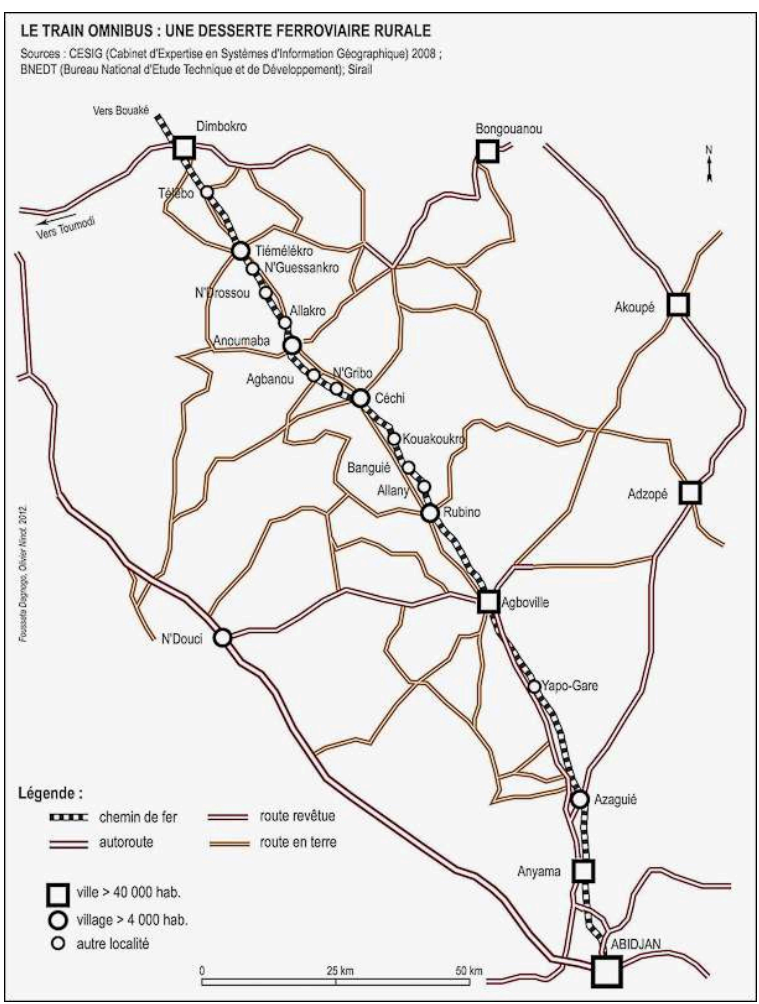

Source : CESIG, 2008 ; BNEDT, Sitarail. Réalisation : F. Dagnogo, O. Ninot, 2012

\section{Abandon des dessertes rurales et aggravation de l'enclavement}

La suppression du train omnibus a complètement déstabilisé le réseau marchand qui en dépendait car les gares situées entre Agboville et Dimbokro ne sont désormais plus desservies par le train. Seuls les trains express $n^{\circ} 11$ (d'Abidjan vers Ouagadougou) et 12 (de Ouagadougou vers Abidjan) offrent encore un service de transport mixte de produits et de voyageurs. Mais au-delà de Dimbokro, l'express $n^{\circ} 12$ ne s'arrête qu'à Agboville; l'express $\mathrm{n}^{\circ} 11$ (en direction du Burkina Faso) ne s'arrête que 2 minutes à Céchi, juste le temps de laisser descendre les commerçantes revenant d'Abidjan qui ont exercé une forte pression sur la direction de la Sitarail pour obtenir ce temps d'arrêt. En effet, ces commerçantes, au retour d'Abidjan avec les revenus de la vente de leurs produits vivriers, sont exposées sur la route à un risque important de braquage. La possibilité de prendre le train est donc pour elles un facteur important de sécurité.

Car l'enclavement dans lequel se trouvent plongés les villages et les bourgs autrefois desservis par l'omnibus et le coup d'arrêt porté au commerce qui s'y greffait tiennent aussi à la faiblesse de l'offre de transport routier. En effet, la route qui relie Agboville à ces localités est si mauvaise qu'elle n'est que peu fréquentée. Les transports, quand ils sont possibles, y sont particulièrement onéreux. Du coup, Agboville n'a pas vu non plus son aire d'influence se renforcer avec la suppression des gares rurales, le transport routier s'étant montré incapable de prendre le relai du rail pour le transport de produits vivriers.

Plus au nord, Boli et Raviart ont subi les mêmes conséquences que les localités situées sur la ligne de l'omnibus. En effet, après la crise post électorale en 2010, la Sitarail a 
arrêté la desserte de ces villages en transformant leur gare en gare temporaire et donc facultative. Depuis, les productrices d'attiéké 8 sont obligées d'aller, par la route, jusqu'à Bouaké (située à $82 \mathrm{~km}$ de Boli et à $60 \mathrm{~km}$ de Raviart) pour envoyer leur production au Burkina Faso. Le prix du transport ayant doublé ${ }^{9}$ depuis la fermeture de la gare ferroviaire, les petites productrices d'attiéké se sont trouvées exclues de ce commerce car elles n'ont pu supporter cette augmentation ni trouver dans la location de camionnettes (trop chère également) une solution alternative. La suppression des arrêts à Boli et à Raviart a finalement eu deux effets. D'une part, elle a réduit le nombre de commerçantes de la filière attiéké ; d'autre part, elle a contribué à renforcer la centralité des gares de Bouaké et de Dimbokro, vers lesquelles convergent les produits. Pourtant, l'arrêt des trains express dans ces gares avait contribué à dynamiser l'économie des deux localités spécialisés dans la production et la vente de l'attiéké. Selon nos enquêtes de terrain en 2010, les productrices des deux localités pouvaient, à chaque voyage, charger ensemble un demi-wagon de sacs d'attiéké de 50 à 60 kilos. Par exemple à Boli, 52 femmes recevaient des commandes de 10 à 50 sacs de la part des commerçantes du Burkina Faso à chaque passage de train.

Depuis la privatisation, le chemin de fer Abidjan-Niger a donc progressivement perdu son rôle d'axe sur lequel se greffait une partie de l'économie vivrière. La politique de sélection des gares du transport de voyageurs par la Sitarail au nom de la rentabilité financière a été à la base de l'isolement de plusieurs localités ivoiriennes. C'est le cas par exemple des localités situées au centre de la Côte d'Ivoire dans la vallée du Bandama, dans le département de Katiola, et rattachées à la sous-préfecture de Niakaramadougou, situées à $20 \mathrm{~km}$. Difficiles d'accès en raison de l'état de dégradation du réseau routier qui les relie entre elles et à la route nationale pourtant proche, Loho, Tiengala, Kiohan, Niangbo, Niédiékaha, sont ainsi victimes d'un effet tunnel (Offner, Pumain, 1996), depuis l'abandon des dessertes ferroviaires (carte 4). En l'absence de services de transports routiers efficaces, les habitants de ces localités n'ont pas la possibilité de se rendre facilement dans d'autres gares, plus éloignées, pour continuer à échanger avec les voyageurs et les commerçants du train. La suppression de l'arrêt du train est vécue ici comme un véritable traumatisme. D'autant que la Sitarail n'a pas associé les populations rurales à ses choix et n'a pas pris en compte l'importance de la desserte de ces multiples localités dans la vie quotidienne et pour les économies locales. Elle a par ailleurs mené cette politique à une période où l'État, considérablement affaibli par les crises politiques, n'a pas pu jouer pleinement son rôle dans l'organisation de son territoire et dans la politique de transport national. Sitarail s'est retrouvée sans interlocuteur de poids qui puisse imposer le respect des engagements relatifs au maintien d'un service public de transport de voyageurs. 
Carte 3 - Fermeture des gares rurales et enclavement dans le département de Katiola

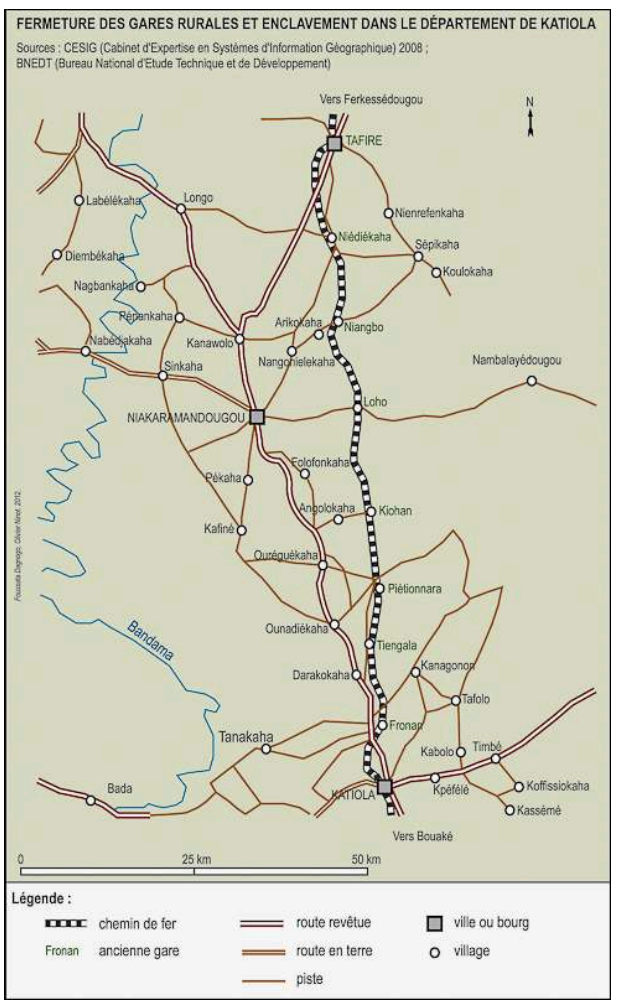

Source : CESIG, 2008 ; BNEDT. Réalisation : F. Dagnogo, O. Ninot, 2012.

\section{Conclusion}

Sans pour autant sous-estimer la portée de la redynamisation et de la sécurisation du trafic de marchandises à longue distance (dont celui de transit à destination des pays enclavés) réussies par Sitrail, cet article révèle l'autre face, moins brillante, de la privatisation du chemin de fer ivoiro-burkinabé. En effet, l'accroissement des tonnages transportés masque les effets néfastes de la privatisation sur les localités traversées mais aussi les doutes qu'il est possible d'émettre sur l'avenir du chemin de fer, dont l'infrastructure est vieillissante. La Sitarail, comme le stipule la convention de concession, est techniquement et financièrement responsable de l'exploitation des services de marchandises et de voyageurs, de l'entretien du matériel, de la gestion courante du domaine ferroviaire et en partie du renouvellement et l'aménagement des infrastructures ferroviaires. Pourtant, depuis 1995, très peu d'investissements ont été accordés au chemin de fer "Abidjan-Niger», malgré l'accroissement du trafic de marchandises. Surtout, à travers la politique de sélection des gares, la Sitarail a aggravé la situation d'enclavement des localités rurales (Loho, Boli, Raviart, etc.), générant des effets tunnels préjudiciables aux économies locales. Le groupe Bolloré, actionnaire majoritaire de la Sitarail et également présent dans l'ensemble de la chaîne de transport, semble, seul, tirer profit de cette infrastructure pour maitriser l'acheminement des marchandises de «bout en bout ». Comme pour d'autres chemins de fer africains privatisés, la réduction importante des services de transport de voyageurs sur l' "Abidjan-Niger", les désaccords entre États et société privée exploitante relatifs notamment au financement de l'entretien du matériel, témoignent 
des divergences persistantes des intérêts privés et des attentes publiques dans le régime concessionnaire (Proparco, 2011).

Après avoir joué un rôle important dans la mise en valeur coloniale et dans l'essor de plusieurs localités, le chemin de fer apparait aujourd'hui en Côte d'Ivoire comme une infrastructure extraterritoriale destinée à désenclaver les pays sans débouchés maritimes. À ce titre, l'étude de ce corridor de transport international ouest africain depuis un pays côtier confirme les résultats mis en évidence par d'autres travaux menés dans des États sahéliens (Lombard, Ninot, 2012): les projets logistiques des grands groupes internationaux s'éloignent des projets de développement national et local à mesure que leur autonomie progresse sur ces corridors (par le jeu des privatisations, des concessions d'exploitation, etc.). Pourtant, entre la situation initiale où le chemin de fer desservait un nombre important de localités rurales et celle où seules les principales villes sont desservies, une voie intermédiaire était sans doute possible. Le maintien d'une desserte pour un certain nombre de gares sélectionnées aurait pu servir les intérêts locaux et renforcer la hiérarchie urbaine au niveau national. Les raisons financières et la stratégie du groupe Bolloré ont primé sur cette possible option. De ce fait, cette évolution interroge, de façon plus générale, fortement sur les politiques de privatisation prônées par les institutions internationales en Afrique subsaharienne, qui semblent loin de fournir la solution à tous les maux nés des dysfonctionnements du chemin de fer. L'exemple de la ligne «Abidjan-Niger » met au contraire en évidence les contradictions entre les politiques de privatisation et celles de développement, qui nécessitent une prise en compte de toutes les couches sociales et de tous les espaces.

\section{BIBLIOGRAPHIE}

Aloko N.-J., 1989. Transport, communication et organisation de l'espace en Côte d'Ivoire, Thèse de doctorat d'État, Université d'Abidjan, 1262 p.

Bavoux J.-J., Beaucire F., Chapelon L., Zembri P., 2005. Géographie des transports. Armand Colin, $231 \mathrm{p}$.

Bredeloup S., 1989. Négociants du long cours : rôle du commerce dans une région de la Côte d'Ivoire en déclin. Paris, l'Harmattan, 319 p.

Chaléard J.-L., 2006. Le rôle du chemin de fer dans le sud-est de la Côte d'Ivoire. Le cas du département d'Agboville. In Chaléard J.-L., Chanson-Jabeur C., Béranger C., Le chemin de fer en Afrique, Paris, Karthala, PRODIG et SEDET, p. 87-104.

Chaléard J.-L., Chanson-Jabeur C., Béranger C., 2006. Le chemin de fer en Afrique, Paris, Karthala, PRODIG et SEDET, $401 \mathrm{p}$.

Debrie J., De Guio S., 2004. Interfaces portuaires et compositions spatiales : instabilités africaines. Autrepart, 2004/4, n 32, p. 21-36. 
Debrie J., 2003. Transport et privatisation dans l'ouest-africain. L'information géographique, Volume 67-2/2003, SEDES, p. 125-134.

Lombard J., Ninot O., 2010. Connecter et intégrer : les territoires et les mutations des transports en Afrique. BAGF 2010-1, p. 69-85.

Lombard J., Ninot O., 2012. Des axes et des pôles. Corridors ouest-africains et développement territorial. Géotransports, à paraître. $14 \mathrm{p}$.

Lombard J., Steck B. (dir.), 2004. Transports aux sud : pouvoirs, lieux et liens. Autrepart $n^{\circ} 32$, Armand Colin, IRD, 203 p.

Maestri E., 1976. Le chemin de fer en Côte d'Ivoire : Historique, problème techniques, influences sociales, économiques et culturels. Thèse de doctorat, Université de Provence, $870 \mathrm{p}$.

Martin B., Micoud M., 1998. Ajustement structurel et privatisation des chemins de fer. Politique de la Banque Mondiale et pratiques gouvernementales en Côte d'Ivoire et au Ghana. Londres, Public World, 12 p, http://www.publicworld.org/files/warailfrench.pdf

Offner J.-M., 1993. Les effets structurants du transport : mythe politique, mystification scientifique. L'espace géographique $\mathrm{n}^{\circ} 3$, p. 233-242.

Offner J.-M., Pumain D., 1996. Réseaux et territoires, significations croisées. La Tour d'Aigues, Editions de l'Aube, $284 \mathrm{p}$.

Peguy P-Y, 1999. Les performances des entreprises ferroviaires. In Let-Inrets, Actes du STRASS 5: Les projets sectoriels des transports en Afrique Sub-Saharienne, Bilan et réflexions, p. 235-245.

PROPARCO, 2011. Quel rôle du secteur privé dans le développement du rail africain ?. Secteur privé et développement, $\mathrm{n}^{\circ} 09 / \mathrm{mars} 2011,28 \mathrm{p}$.

République de Côte d'Ivoire, Direction et Contrôle des Grands Travaux, 1988. Plan national de transport - Rapport final 4: Transport ferroviaire. $83 \mathrm{p}+$ annexes.

République de Côte d'Ivoire, Institut National de la Statistique, 2001. Recensement Général de la Population et de l'Habitat 1998.

SITRAIL, 2011. Livret de la marche des trains 2011. 30 p.

SITRAIL, 2009. Nouvelles du réseau, bulletin d'information de SITARAIL. N¹52-153/154-155, septembre/novembre-décembre 2009, 38 p.

SITRAIL, 2006. Nouvelles du réseau, bulletin d'information de SITARAIL. $\mathrm{N}^{\circ} 110-111$, mars-avril 2006, $23 \mathrm{p}$.

SITRAIL, 2004. Nouvelles du réseau, bulletin d'information de SITARAIL. N86-87, mars-avril, 2004, Spécial Anniversaire du Réseau, $70 \mathrm{p}$.

Steck B., 2009. Transport et développement. In Brocard M. (sous la dir. de), Transports et territoires, enjeux et débats, collection Carrefours, Ellipses : Paris, 188 p., p. 125-156.

Tape Bidi J., 2010. Quelques réflexions sur l'existence d'un port sec à Bouaké en Côte d'Ivoire. In Fodouop K., Tape Bidi J., L'armature du développement en Afrique, Karthala. 264 p.

Yoboue A. et al, 1996. Étude de Marché sur le transport de voyageurs. SITARAIL, 49 p. + annexes. 


\section{NOTES}

1. Ce chemin de fer, comme celui qui part de Dakar, avait pour vocation première de relier le vaste bassin du Niger, cœur continental du projet colonial français en Afrique de l'ouest, aux côtes atlantiques.

2. Des propos recueillis pendant les enquêtes de terrain (F. Dagnogo, 2008 et 2010) auprès des responsables des sociétés de patrimoine ferroviaire de Côte d'Ivoire et du Burkina Faso.

3. Nouvelles du réseau : revue du bulletin d'information de la Sitarail, n86-87, mars-avril 2004.

4. Avec plus d'un million de tonnes en 2001, la Sitarail a dépassé le record de 900000 tonnes établi par la R.A.N. en 1973.

5. D'après nos enquêtes de terrain de 2008 et 2010.

6. Selon un responsable de la Sitarail que nous avons interrogé.

7. Appelées communément «femmes du train omnibus ».

8. Semoule de manioc cuite à la vapeur, une nourriture très prisée en Côte d'Ivoire et au Burkina Faso.

9. Le transport jusqu'à Bouaké par la route coûte $2550 \mathrm{~F}$ CFA par sac alors que les frais de transport par train de Bouaké au Burkina Faso sont de 2500 F CFA.

\section{RÉSUMÉS}

Le chemin de fer Abidjan-Niger a joué un rôle important dans l'économie coloniale. Après une gestion publique à partir de 1960 qui a conduit à son déclin, son exploitation a été concédée en 1995 sous la pression des bailleurs de fonds à la Sitarail (Société Internationale des Transports par rail). Si, depuis lors, le trafic de marchandises a connu une croissance soutenue, la suppression des gares rurales par la Sitarail dans un souci de rentabilité économique a eu pour conséquence la marginalisation de plusieurs petites localités. À partir de données quantitatives et qualitatives collectées entre 2008 et 2010 , cet article essaie de montrer que le chemin de fer Abidjan-Niger dans le contexte de gestion privée et de tension politique persistante entre le nord et le sud du pays se présente davantage comme un corridor de désenclavement des pays sans débouché maritime que comme un axe structurant le territoire ivoirien.

\section{INDEX}

Mots-clés : chemin de fer, Côte d'Ivoire, enclavement, extraterritorialité, organisation de l'espace, privatisation, Sitarail

Thèmes : Sur le Champ - Sur le Terrain

\section{AUTEURS}

\section{FOUSSATA DAGNOGO}

Foussata Dagnogo, fouss105@yahoo.fr, est Doctorante en géographie, Université de Paris 1, UMR PRODIG. 


\section{OLIVIER NINOT}

Olivier Ninot, olivier.ninot@prodig.cnrs.fr, est ingénieur de recherche CNRS, membre de l'UMR PRODIG. . Avec Jérôme Lombard, il a participé en 2010 à la journée sur les cinquante ans des indépendances africaines, organisée par l'Association des géographes français ( Connecter et intégrer. Les territoires et les mutations des transports en Afrique », Bulletin de l'Association des géographes français, 2010,1) et dirigé le numéro spécial d'Espaces Populations Sociétés consacré aux nouvelles mobilités dans les Suds (http://eps.revues.org/index4001.htm).Il a co-publié :

- Magrin G., Ninot O., Cesaro J.D., 2011. L'élevage pastoral au Sénégal : entre pressions spatiales et mutations commerciales. Mappemonde, $\mathrm{n}^{\circ} 101,2011-1,17 \mathrm{p}$.

- Cesaro J.D., Magrin G., Ninot O. 2010. Atlas de l'élevage au Sénégal. Paris, Prodig, 32 p. http:// hildegarde.univ-paris1.fr/elevage-senegal/

\section{JEAN-LOUIS CHALÉARD}

Jean-Louis Chaléard, est Professeur des Universités, Université de Paris 1 et membre de l'UMR PRODIG. 